\section{De la Cruz-Campos SB ${ }^{1}$ Cárdenas-Flores $\mathrm{CM}^{2}$}

${ }^{1} \mathrm{CD}$, Residente Especialidad de Odontopediatría UCSUR.

2 CD,Esp,Mg. Odontopediatría, Docente Odontopediatría UCSUR.

\section{REPORTE} DE CASO

\title{
USO DE PLACAS PALATINAS PARA MEJORAR EL CIERRE BUCAL Y LA POSICIÓN LINGUAL EN PACIENTES CON SÍNDROME DE DOWN: RELATO DE CASO CLÍNICO
}

\section{RESUMEN}

OBJETIVO: El propósito de presentar este artículo es reportar un caso clínico donde se trata de mejorar la posición lingual, el cierre bucal mediante el uso de la placa palatina en una paciente con Síndrome de Down.

DESCRIPCIÓN: Paciente de 3 años 10 meses con Síndrome de Down, con hipotonía de músculos orofaciales, boca abierta y labio inferior evertido, se coloca una placa palatina para mejorar el cierre bucal y el posicionamiento de la lengua, después de 4 meses de uso se logró mejorar la posición lingual y el cierre bucal.

CONCLUSIONES: El uso de la placa palatina mejora el cierre bucal por estimulación de los músculos periorales y mejora la posición de la lengua por estimulo del botón lingual de la placa.

PALABRAS CLAVE: Placas palatinas, Síndrome de Down, Cierre bucal, Posición lingual.

PALATINE PLATE USE FOR IMPROVING THE CLOSING MOUTH AND TONGUE POSITION IN PATIENTS WITH DOWN SYNDROME: CLINICAL CASE REPORT

\section{ABSTRACT}

OBJECTIVE: The purpose of this article is to present a case report where it comes to improving the position lingual, oral closure by using the palatine plate in a patient with Down syndrome.

DESCRIPTION: Patient 3 years 10 months with Down Syndrome, with hypotony of orofacial muscles, open mouth and lower lip everted a palatine plate is positioned to improve oral closure and positioning of the tongue, after 4 months of use he managed to improve oral tongue position and close.

CONCLUSIONS: The use of palatine plate improves oral stimulation closure of the perioral muscles and improves the position of the tongue lingual stimulus button plate.

KEY WORDS: Palatal plates, Down syndrome, oral closure, lingual position. 


\section{INTRODUCCIÓN}

El Síndrome Down (SD) es uno de los trastornos genéticos humanos más frecuentes, aunque en el Perú no contamos con cifras exactas sobre la cantidad de personas afectadas, según la Sociedad Peruana de Síndrome Down serían alrededor de 80, 000 personas. $^{1}$

Este síndrome se origina por la presencia de un cromosoma 21 extra que clínicamente presenta numerosos problemas asociados, incluidos los problemas estomatológicos, siendo uno de los principales problemas la marcada hipotonía muscular general la que dificulta las funciones básicas; y a nivel orofacial genera una expresión característica de boca abierta, lengua hipotónica descansando sobre el labio inferior y gran salivación ${ }^{2}$. Los niños con SD presentan disminución de las dimensiones del maxilar con consecuente hipoplasia del tercio medio facial, hipotonía y protrusión de la lengua con dificultad en el cierre labial. Estas características influyen en el desarrollo cráneo facial con repercusiones oclusales. ${ }^{3}$

La hipotonía muscular en el Síndrome Down, tiene como origen las características anatomopatológicas del cerebro, en donde encontramos menos neuronas y menos sinapsis entre ellas, provocando un déficit neurológico global, que causa una excesiva flexibilidad de los ligamentos, ocasionando déficit en los procesos motrices como hablar, caminar o gatear. ${ }^{4}$ Por tanto, la cavidad oral de estos pacientes se ve afectada tanto en sus estructuras, como en sus funciones, provocando la marcada hipotonía, impidiendo o dificultando algunas funciones básicas del ser humano tales como la masticación, la deglución, la fonación, entre otras. Este hecho limita el desarrollo del lenguaje oral, principalmente la articulación, afectando su comunicación, la inclusión escolar, social y su calidad de vida (Fiske y Shalik, 2001). ${ }^{3}$

En la década del 70 el médico argentino Castillo-Morales, introdujo en Europa un método para la rehabilitación neuromotora de niños con Síndrome Down. Este incluye una terapia de re- gulación orofacial, la que está conformada por ejercicios de estimulación orofacial, sumada a un dispositivo para el entrenamiento de la lengua y los músculos peribucales: La Placa Palatina (PP), la cual mejora la función orofacial. ${ }^{2,5} \mathrm{La}$ PP es un dispositivo intraoral que posee un botón estimulador para mejorar la posición de la lengua y otros botones estimuladores para el labio superior que inducen el cierre labial y posicionamiento adecuado de la lengua con mejoría de la musculatura orofacial en especial la función del orbicular de los labios y músculos de la mímica, que conduce a mejoras en la succión, la articulación, deglución y la respiración nasal y consecuentemente un desarrollo orofacial más armónico y una mejora en su pariencia. ${ }^{1,6}$ Castillo Morales recomendó su uso en niños entre 2 meses y 2 años debido a que en esta etapa se dan la mayoría de cambios neurológicos en el niño. El éxito de este tratamiento depende siempre del nivel de cooperación del paciente, y en el caso de los niños con Síndrome Down, depende de la cooperación y participación de los padres. ${ }^{7}$

En un estudio piloto realizado en Chile se observó los efectos de las placas palatinas estimulantes y de los ejercicios orofaciales en la función y tonicidad muscular en niños con Síndrome Down, produciéndose un aumento del cierre bucal y una disminución de la protrusión lingual además de una mejora en la expresión facial. ${ }^{2,8}$ Se han reportado también seguimientos de casos tratados con este método con resultados satisfactorios y se reporta que estas condiciones se mantienen estables, a lo largo del tiempo, después del uso de la PP. ${ }^{9}$

\section{PRESENTACIÓN DEL CASO:}

Se presenta a la consulta una paciente de sexo femenino de 3 años 11 meses de edad con Síndrome Down, sin presencia de ninguna enfermedad sistémica actual, pero refiere diabetes como antecedentes familiares. Al examen clínico extraoral se observa a la paciente con hipotonía marcada de los músculos orofaciales, boca abierta, babeo 
constante, lengua protráctil y labio inferior evertido característicos de su condición. (Fig.1-2)

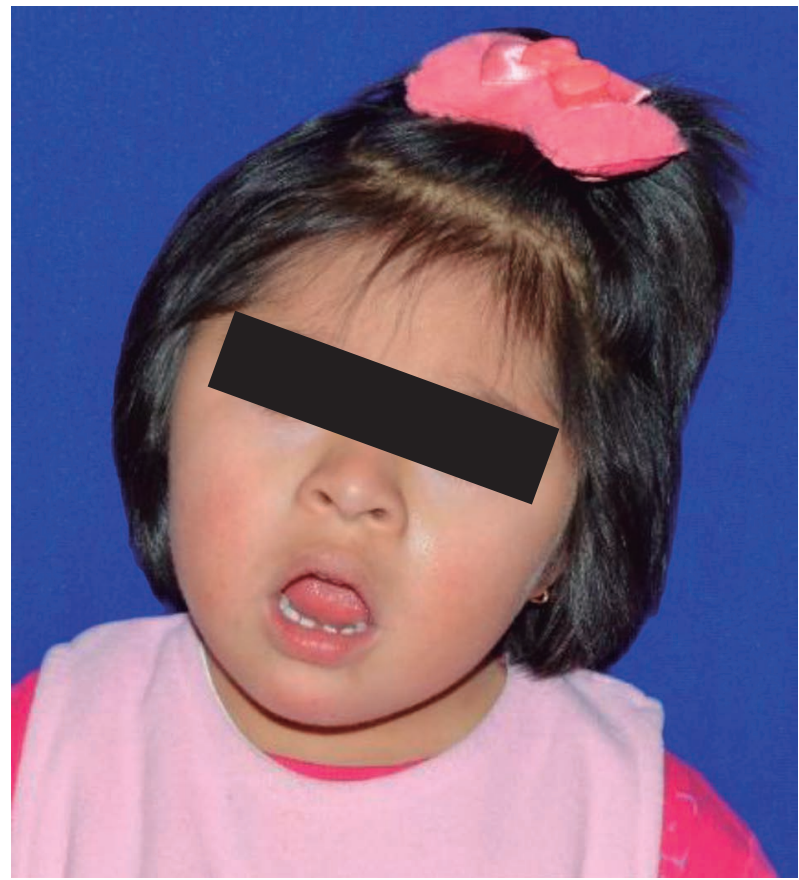

Figura 1. Vista frontal de la paciente.

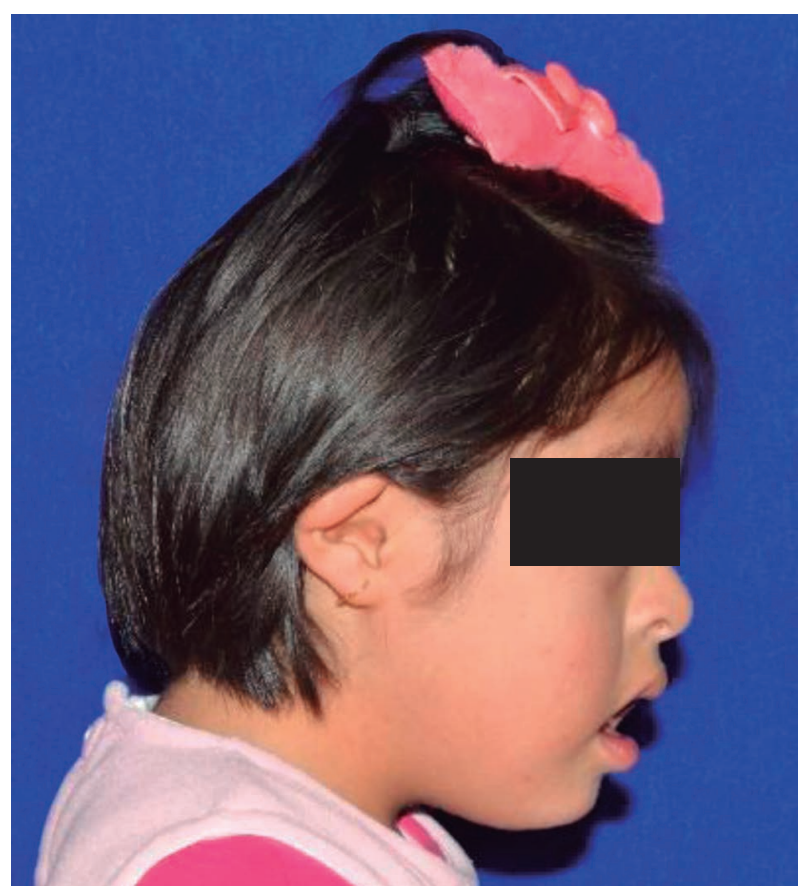

Figura 2. Vista lateral de la paciente.

Al examen intraoral se observa el frenillo lingual laxo, los dientes apiñados en el maxilar superior, agenesia de la pieza 7.2 y maxilar inferior espaciado, fusión de las piezas 8.2 y 8.3. (Fig. 3,4,5)

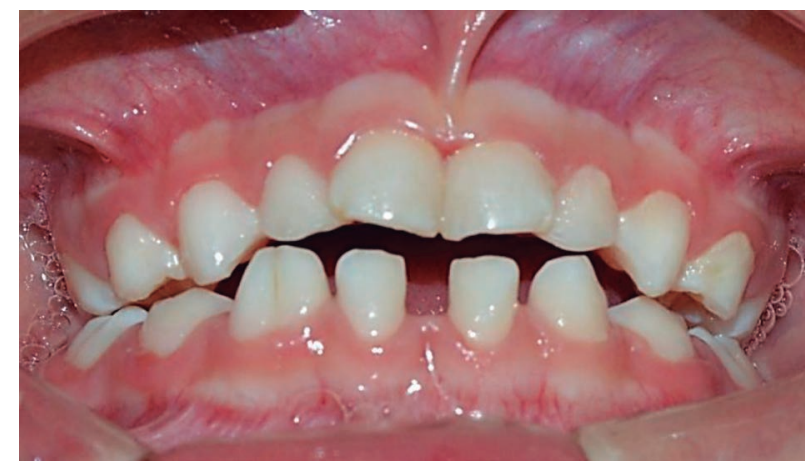

Figura 3. Vista intraoral frontal.

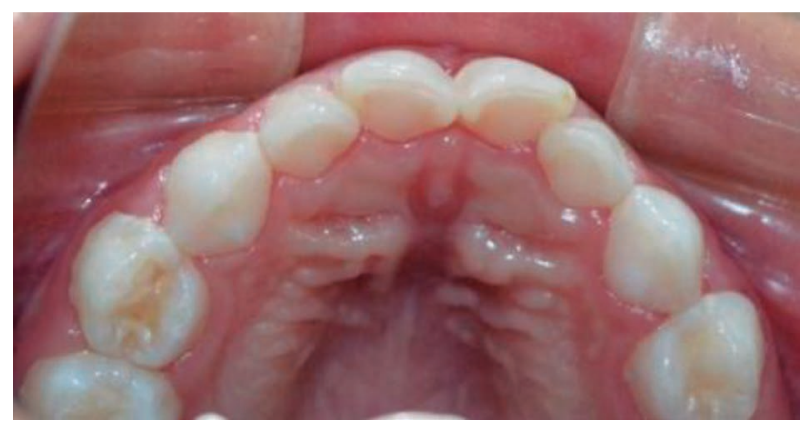

Figura 4. Vista intraoral maxilar superior.

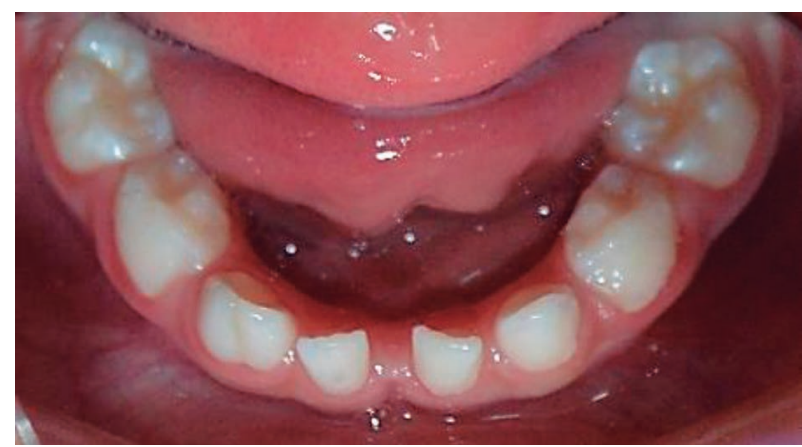

Figura 5. Vista intraoral maxilar inferior.

Después de la evaluación se decidió colocar una PP para mejorar el cierre bucal y el posicionamiento de la lengua. Se tomaron las impresiones con alginato para la confección de los modelos de trabajo. Luego se procedió al diseño de la placa siguiendo las especificaciones del método Castillo Morales, una placa mucodento soportada de acrílico con retenedores de punto, con un botón estimulador palatino y botones estimuladores vestibulares para los músculos del labio superior. (Fig. 6,7,8) 


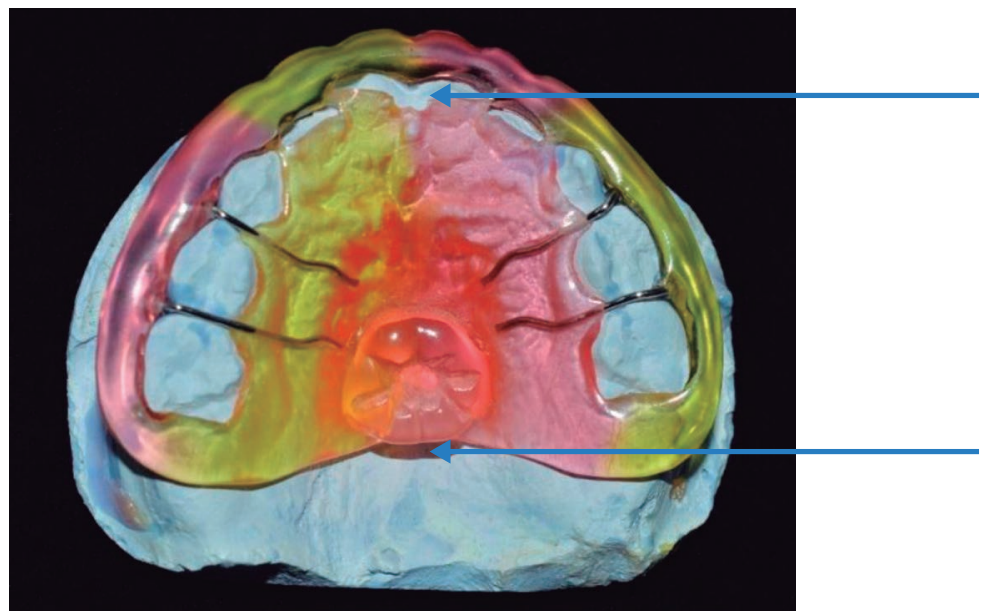

\author{
Botones \\ estimuladores \\ del labio
}

\title{
Botón \\ estimulador \\ de la lengua
}

Figura 6. Placa palatina en acrílico.

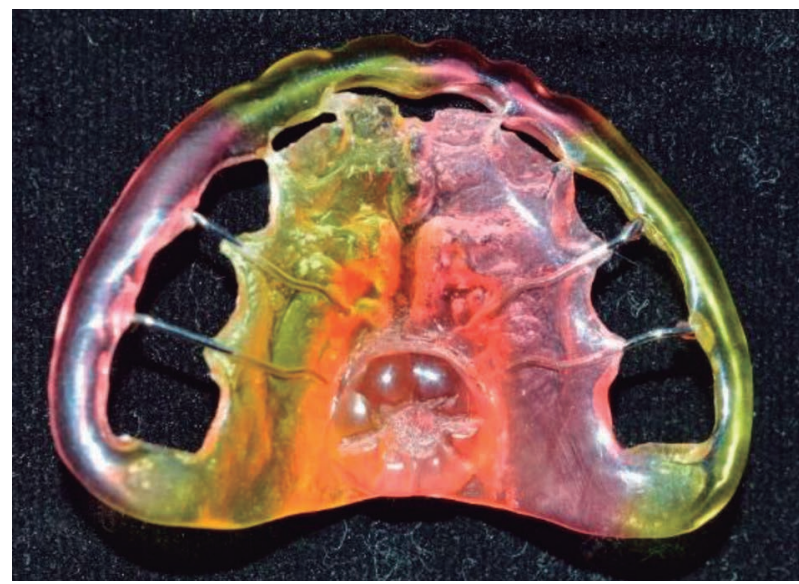

Figura 7. Placa palatina vista palatina.

Luego se instaló la placa en boca indicando a la madre que esta debía ser usada por espacio de 15 a 20 minutos de 4 a 5 veces al día durante los primeros quince días como etapa de adaptación lue-

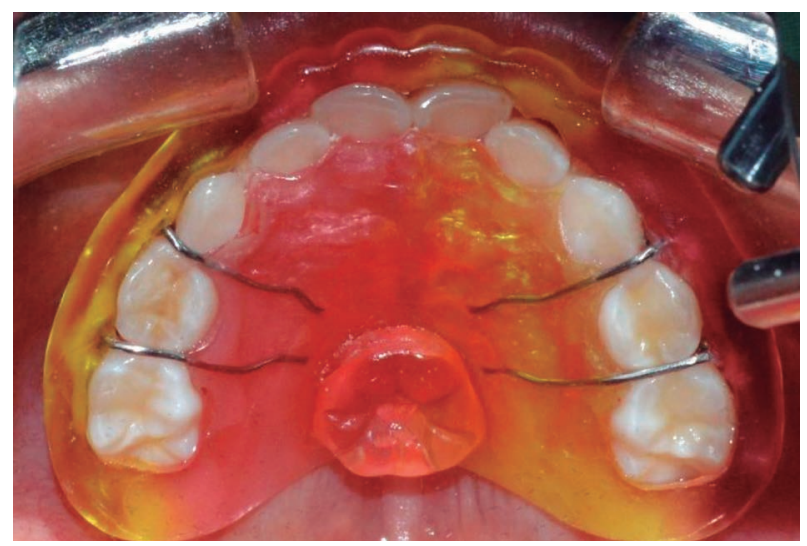

Figura 9. Instalación de PP vista oclusal.

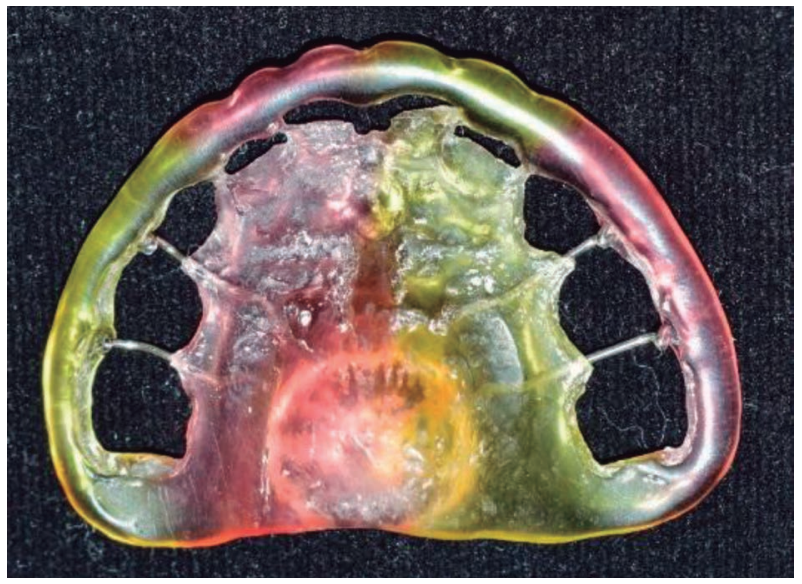

Figura 8. Placa palatina vista mucosa.

go este tiempo debía de ir aumentado hasta llegar a usar la placa de manera permanente retirándola solo para comer y dormir. Los controles debían ser periódicos cada 15 días. (Fig. 9.10)

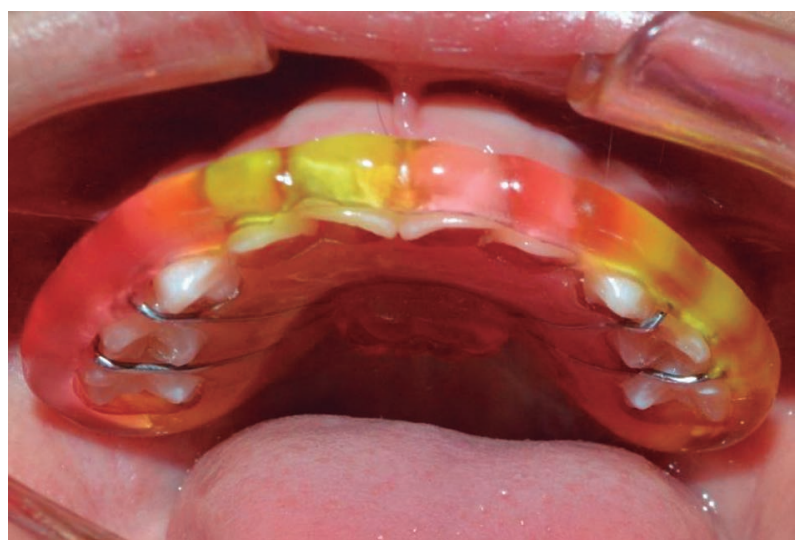

Figura 10. Instalación de PP vista frontal. 
Después de la primera quincena la madre llegó a su primer control y refirió que la paciente no se podía adaptar al uso de la placa debido a que siempre estaba con la boca abierta. Se hizo la reevaluación y se decidió cambiar de ubicación el botón estimulador palatino ubicándolo en una zona más funcional a nivel de las rugas palatinas. (Fig. 11 y 12)

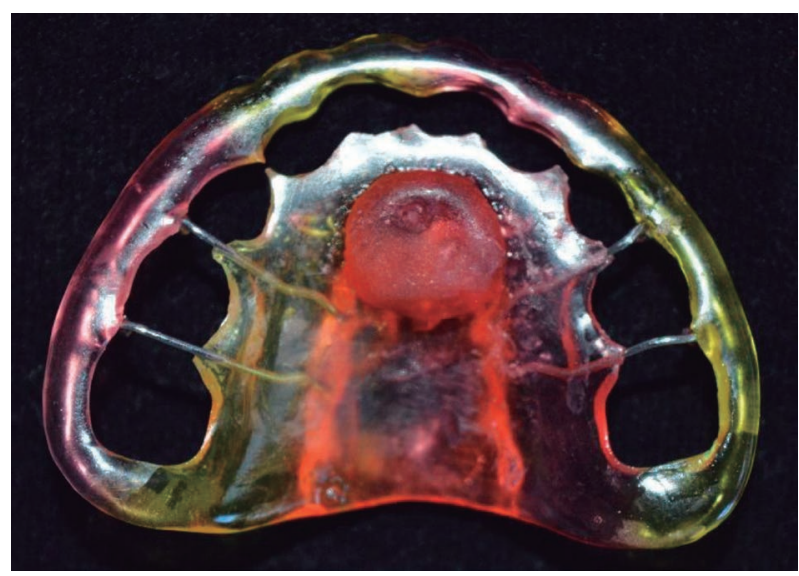

Figura 11. Placa palatina modificada vista palatina.

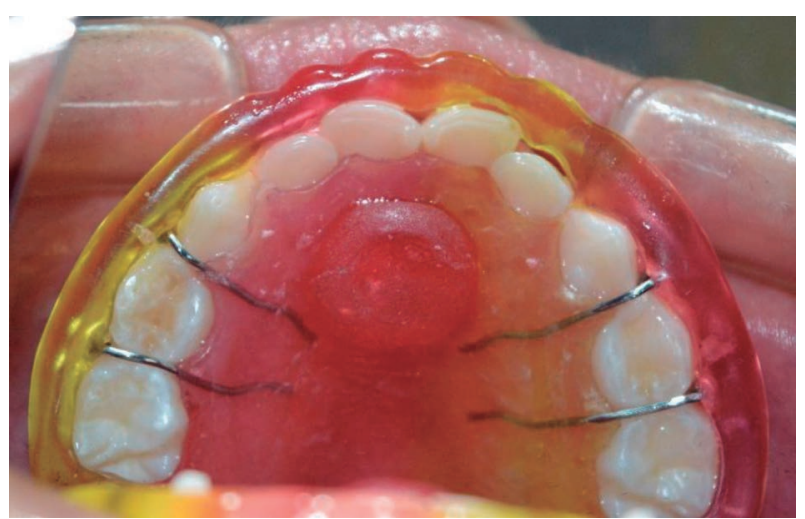

Figura 12. Instalación de PP modificada vista oclusal.

Se volvió a probar la PP en boca con resultados inmediatos de un cierre bucal favorable. Después de 4 meses de uso continuo y sus controles periódicos, se logró mejorar de manera significativa la posición lingual y el cierre bucal. (Fig 13,14)

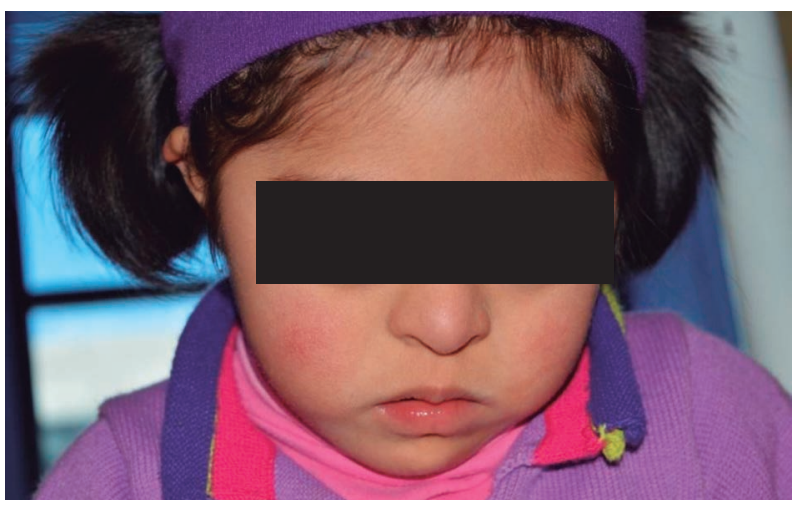

Figura 13. Vista frontal de la paciente 4 meses después del uso de la PP.

\section{DISCUSIÓN:}

La rehabilitación orofacial con PP, de los niños con Síndrome Down fue introducida en la década de 1980. Esta debería comprender atención médica multidisciplinaria proporcionada por los pediatras, genetistas, neurólogos, sicólogos, logopedas y odontólogos también. ${ }^{10}$ Los programas de intervención temprana de nuestro medio solo están orientados a la atención pedagógica, sicológica y de terapia de lenguaje por lo que estos deberían de articularse con servicios de odontopediatría y ortodoncia para tratar a tiempo las deficiencia orales que puedan presentar y optimizar su desarrollo. Independientemente de la dificultad que involucra la evaluación de los cambios en la musculatura orofacial, posición de la lengua y el cierre labial durante y después de la terapia con la PP, la evaluación visual sigue siendo uno de los métodos más importantes de seguir el progreso de la rehabilitación orofacial. ${ }^{11}$ En este caso también se hizo uso de la observación o inspección visual llevando un registro fotográfico secuencial para su reporte.

Según Castillo Morales el uso de la PP debería ser iniciado lo más precozmente posible, generalmente durante el primer año de vida, por ser el período de mayor desarrollo del sistema nervioso central y de la boca. ${ }^{7,12}$ En este caso se optó por colocar la PP pese a la edad de la paciente por lo que se le indicó un uso por más prolongado y con 
un uso de una mayor cantidad de horas durante el día además de tener a favor la colaboración plena de los padres. Por ello y basándonos en los resultados obtenidos vemos que también puede ser usado por niños mayores de 3 años de edad con resultados satisfactorios.

Generalmente en los niños con Síndrome Down ocurre un atraso en la erupción de los dientes deciduos además de presentar un desarrollo óseo más lento, esto favorece al uso de la placa por un tiempo más prolongado. ${ }^{13}$ En este caso la paciente llevó una PP por cuatro meses, luego de una reevaluación se decidió cambiar de PP para evitar restringir el crecimiento maxilar.

El cierre bucal y el mejoramiento de la posición lingual se atribuyen en un primer momento a una "reacción primaria" y al contribuir la placa con una constante estimulación se presentan los cambios a mediano plazo. ${ }^{1}$ La evaluación del tono muscular solo podrá ser evaluada a largo plazo por lo que se debe de hacer un seguimiento a la paciente. Además de complementar con un programa de ejercicios miofaciales de estimulación en el servicio de fonoaudiología o terapia de lenguaje.

El diseño de la PP es muy versátil, en la literatura se hacen referencia a muchas adaptaciones de la misma dependiendo de las necesidades específicas del paciente y como vemos en este caso de la edad del paciente. ${ }^{10}$ En este caso con una paciente de más de 3 años y una deglución madura se decidió cambiar la ubicación del botón estimulador palatino a nivel de las rugas palatinas y contribuyó con la adaptación de la paciente a la PP y así poder usarla la mayor cantidad de tiempo y con cambios muy favorables.

\section{CONCLUSIONES:}

El uso de la placa palatina mejora el cierre bucal bilabial por estimulación de los músculos periorales y mejora la posición de la lengua por estimulación lingual del botón palatino de la placa en pacientes con Síndrome Down con marcada hipotonía de los músculos periorales y la lengua como es el caso de la paciente.

Se recomienda seguir con el uso de la PP conjuntamente con un programa de ejercicios de estimulación orofacial para mejorar la tonicidad de los músculos periorales que deberá ser evaluada a largo plazo. La terapia temprana con el uso de la placa palatina debe ser parte integral del programa de rehabilitación multidisciplinaria de los pacientes con Síndrome Down.

\section{REFERENCIAS \\ BIBLIOGRÁFICAS}

1. Padró M, Barraza E, Brucher C, Concha E, Delgado V. "Efectividad del Uso de Placas Palatinas y de la Estimulación Orofacial en el Desarrollo Oral en Niños con Síndrome de Down”. Rev Chil Pediatr 2010; 81 (1): 46-52.

2. Rondal JA. Atención temprana: comunicación y desarrollo del lenguaje. Rev. Síndrome de Down. 2009; 26:26 - 31.

3. Rondal J. Dificultades del lenguaje en el síndrome de Down: Perspectiva a lo largo de la vida y principios de intervención. . Rev. Síndrome de Down. 2006; 23:120 - 128.

4. Fernández P, Fernández C, Díaz J, Schnake M, Guerra C, Díaz F, et al. Estimulación de la musculatura orofacial en niños con síndrome de Down. Canal Down 21.org [Revista en internet] 2013 Marzo [acceso 6 de Septiembre 2014]; (142). Disponible en: http://www. down21.org/web_n/index.php?option=com content $\&$ view $=$ category $\&$ id $=1205 \% 3$ Aarticulo-profesional\&layout=default\&Itemid=169

5. Carlstedt K, Henningsson G, Mc Allister A, Dahlo G. Long-term effects of palatal plate therapy on oral motor function in children with Down Syndrome evaluated by video registration. Acta Odontol Scan 2001; 59: 63-8.

6. Culebras E, Silvestre J, Silvestre F. Alteraciones odonto-estomatológicas en el niño con Síndrome de Down. Rev Esp Pediatr 2012; 68(6): 434-439. 
7. Carneiro V, Gonzáles J, Fabián F. Utilización de la placa palatina de memoria y desarrollo orofacial en infante con Síndrome de Down. Revista Cubana Estomatología. 2012;49(4):305-311.

8. Nazer J, Cifuentes L. Estudio epidemiológico global del Síndrome Down. Rev Chil Pediatr 2011; 82 (2): 105-112.

9. Molina J. Atención y cuidados odontológicos para los niños con síndrome de Down. Rev Síndrome de Down. 2005;22: 15-19.

10.Matthews T, Cudzilo D, Walaz J, Kawala B. Rehabilitation of the Orofacial Complex by
Means of a Stimulating Plate in Children with Down Syndrome. Adv Clin Exp Med. 2015; 24(2):301-305.

11.Limbrock G, Castillo-Morales R, Hoyer H, Stöver B, Onufer C. The Castillo-Morales approach to orofacial pathology in Down syndrome. Int J Orofacial Myology. 1993;19:30-7.

12.Buzunáriz N, Martínez M. El desarrollo psicomotor en los niños con Síndrome de Down y la intervención de fisioterapia desde la atención temprana. Rev Méd Internacional sobre el Síndrome de Down. 2008; 12(2):28-32.
Recibido: 12-02-2016

Aceptado: 23-03-2016
Correspondencia: Carol Magaly Cárdenas Flores, Silvia De la Cruz Campos

Dirección: caryto2007@hotmail.com 